IPC2018-78278 DRAFT

\title{
THE EFFECT OF MATERIAL STRAIN HARDENING ON THE DETERMINATION OF CTOD R-CURVES USING SENB SPECIMENS
}

\author{
Philippa L Moore \\ TWI Ltd \\ Great Abington, Cambs, UK \\ philippa.moore@twi.co.uk
}

\author{
Wee Liam Khor \\ Brunel University /NSIRC \\ Great Abington, Cambs, UK \\ weeliam.khor@gmail.com
}

\begin{abstract}
In ductile materials the fracture toughness is usually characterised by a tearing resistance curve, or R-curve, plotting the fracture toughness in terms of $\mathrm{J}$ or CTOD against crack extension. Recent research has evaluated the methods to determine CTOD in engineering alloys with a wide range of yield to tensile $(\mathrm{Y} / \mathrm{T})$ ratios for single point CTOD. This work develops the investigation into R-curves, and reviews the assumptions about SENB specimens deforming under rigid rotation, the evaluation of CTOD from $\mathrm{J}$ for R-curves, and the nature of tearing initiation in low $\mathrm{Y} / \mathrm{T}$ ratio stainless steel, from comparisons against a series of silicone replicas cast from the SENB specimen notch during fracture toughness tests.

For CTOD R-curves, the methods based on CTOD from $\mathrm{J}$ in ISO 12135 and ASTM E1820 gave lower and less accurate Rcurves than the rigid rotation methods in BS 7448-4 and WES 1108. However, the accuracy of the BS 7448-4 formula varied for the different strain hardening materials, and overestimated the R-curves in the low tensile ratio stainless steel.

Investigations into the effect of the assumption about rigid rotation in different strain hardening materials led to a rotational factor function of tensile ratio, $r_{p}$ sh, to be developed from numerical modelling. When this function was substituted into standard equations in place of the fixed value of $r_{p}$ an improvement in the accuracy of BS 7448-4 R-curves compared to replica measurements was seen for the range of strain hardening investigated, but it did not significantly improve the accuracy of the WES 1108 formula, which accounts for strain hardening in other parameters.

A combination of the elastic CTOD part of the WES 1108 formula, with the plastic CTOD incorporating the modified rotational factor, was concluded to offer the optimum method to determine CTOD R-curves for a range of strain hardening materials.
\end{abstract}

\section{NOMENCLATURE}

$A_{p}$ Plastic work, area under the plastic component of the load versus displacement graph, $\mathrm{Nmm}$

$\mathrm{a}_{0} \quad$ Original crack length, $\mathrm{mm}$

$\mathrm{a}_{0} / \mathrm{W}$ Crack length to specimen width ratio

$\mathrm{b}_{0} \quad$ Remaining ligament ahead of the crack tip, $\mathrm{W}-\mathrm{a}_{0}, \mathrm{~mm}$

B Specimen thickness, $\mathrm{mm}$

$\mathrm{B}_{\mathrm{N}}$ Net thickness after machining side-grooves, mm

CMOD Crack mouth opening displacement, $\mathrm{mm}$

E Modulus of elasticity, $\mathrm{N} / \mathrm{mm}^{2}$

$\mathrm{J}$ Path independent strain energy around the crack, also called J-integral, $\mathrm{Nmm}^{-1}$

$\mathrm{K}$ Stress intensity factor, $\mathrm{Nmm}^{-3 / 2}$

LLD Load line displacement, $\mathrm{mm}$

$\mathrm{m}$ Factor relating CTOD to $\mathrm{J}$ or $\mathrm{K}$ (sometimes referred to as a "constraint" factor)

$\mathrm{V}_{\mathrm{p}}$ Plastic component of the clip gauge opening displacement, $\mathrm{mm}$

W Specimen width, $\mathrm{mm}$

$\mathrm{z}$ Vertical height above the crack mouth where displacement is measured, $\mathrm{mm}$

$\delta \quad$ Crack tip opening displacement, CTOD, mm

$\delta_{0} \quad$ CTOD from the opening of the original crack tip, $\mathrm{mm}$

$\delta_{45}$ CTOD measured based on the distance between $45^{\circ}$ intercepts from the centreline of a blunted crack tip in FE, $\mathrm{mm}$

$\delta_{\text {el }} \quad$ Elastic component of CTOD, mm

$\delta_{\text {FE }}$ CTOD measured from the mid-specimen thickness of the FE model based on the opening at the original crack tip, $\mathrm{mm}$

$\delta_{\mathrm{FE}}$ corr $\delta \mathrm{FE}$ with applied correction factor for tensile ratio validated to experimental results, $\mathrm{mm}$

$\delta_{\mathrm{pl}} \quad$ Plastic component of CTOD, mm

$\delta_{\text {SRC }}$ CTOD measured from the middle thickness of the silicone replica based on the original crack tip, $\mathrm{mm}$ 
$\sigma_{\text {uts }}$ Ultimate tensile stress, $\mathrm{N} / \mathrm{mm}^{2}$

$\sigma_{\mathrm{y}} \quad$ Flow stress defined as $\left(\sigma_{\mathrm{ys}}+\sigma_{\mathrm{uts}}\right) / 2, \mathrm{~N} / \mathrm{mm}^{2}$

$\sigma_{\mathrm{ys}} \quad 0.2 \%$ yield/ proof stress, $\mathrm{N} / \mathrm{mm}^{2}$

$\sigma_{\mathrm{ys}} / \sigma_{\text {uts }}$ Tensile ratio

$\eta_{\mathrm{pl}}$ Geometrical based calibration factor for $\mathrm{J}$

$v$ Poisson's ratio

\section{INTRODUCTION}

\section{CTOD R-curve formulae from current Standards}

In ductile materials it is recommended that the fracture toughness instead of a single value calculated at maximum load, is characterised by a tearing resistance curve (R-curve), which can be defined in terms of CTOD or J. R-curves are included within standards ISO 12135 and ASTM E1820, and BS 7448-4 [1-3]. There is often a modification to the equivalent single point equations to account for crack extension when defining the toughness by plotting an R-curve of CTOD against the increase in crack length, $\Delta \mathrm{a}$. For example, the CTOD equation used in BS 7448-4 is primarily based on the fixed rotational point assumption for single point tests given in BS 7448-1, but with additional $\Delta$ a terms;

$$
\delta_{(\mathrm{i})}=\frac{\mathrm{K}^{2}\left(1-\mathrm{v}^{2}\right)}{2 \mathrm{E} \sigma_{\mathrm{ys}}}+\frac{0.6 \Delta \mathrm{a}+0.4\left(\mathrm{~W}-\mathrm{a}_{0}\right)}{0.6\left(\mathrm{a}_{0}+\Delta \mathrm{a}\right)+0.4 \mathrm{~W}+\mathrm{z}} \times \mathrm{V}_{\mathrm{p}}
$$

The ASTM E1820 method converts CTOD from J calculated iteratively, with each point on the R-curve having a correction factor for crack extension. The conversion is described as;

$$
\delta_{(\mathrm{i})}=\frac{\mathrm{J}_{(\mathrm{i})}}{\mathrm{m} \sigma_{\mathrm{y}}}
$$

Where $J_{(i)}$ is the addition of the instantaneous elastic and plastic J components,

$$
\mathrm{J}_{(\mathrm{i})}=\mathrm{J}_{\mathrm{el}(\mathrm{i})}+\mathrm{J}_{\mathrm{pl}(\mathrm{i})}
$$

The instantaneous elastic $\mathrm{J}$ is similar to that used to calculate single point $\mathrm{J}$, described as;

$$
\mathrm{Jel}_{\mathrm{el}}=\frac{\mathrm{K}_{(\mathrm{i})}{ }^{2}\left(1-\mathrm{v}^{2}\right)}{\mathrm{E}}
$$

The crack extension correction is applied on the plastic $\mathrm{J}$ component [4];

$$
\begin{gathered}
J_{p l(i)}=\left[J_{p l(i-1)}+\left(\frac{\eta_{p l(i-1)}}{b_{(i-1)}}\right)\left(\frac{A_{p l(i)}-A_{p l(i-1)}}{B_{N}}\right)\right] \times[1- \\
\left.\gamma_{p l(i-1)}\left(\frac{a_{(i)}-a_{(i-1)}}{B_{0(i-1)}}\right)\right]
\end{gathered}
$$

Where;

$$
\gamma_{\mathrm{pl}}=0.131+2.131\left(\mathrm{a}_{(\mathrm{i}-1)} / \mathrm{W}\right)-1.465\left(\mathrm{a}_{\mathrm{(i-1})} / \mathrm{W}\right)^{2} \quad \text { Eq.6 }
$$

Where (i-1) and (i) refer to successive loading point increments, for example unload-reload points in the unloading compliance method.

ISO 12135 in the 2016 version dropped the rigid rotation method given in Eq 1 which was in the 2002 version, and instead adopted the $\mathrm{J}$ conversion method for the determination of CTOD for tearing resistance curves. The basic $\mathrm{J}$ equation is the same as used in ASTM E1820, except for the methods to determine the plastic component of J: ISO only allow the plastic work, $A_{p}$ to be determined based on the load-line displacement, LLD, whereas ASTM allows plastic work to be determined from both CMOD and load-line displacement, but the difference between these two approaches is minimal [5], and $\mathrm{J}$ calculated using CMOD is considered to be equivalent to J calculated using LLD. However, a main difference is that ISO uses a different crack extension factor to that in ASTM (Eq. 5). The plastic J used in ISO for Rcurves is shown below;

$$
\mathrm{J}_{\mathrm{pl}(\mathrm{i})}=\frac{1.9 \mathrm{~A}_{\mathrm{p}}}{\mathrm{B}_{\mathrm{N}} \mathrm{B}_{0}} \times\left(1-\frac{\Delta \mathrm{a}}{2 \mathrm{~B}_{0}}\right)
$$

\section{Rigid rotation assumption for CTOD determination}

The definition of CTOD is the displacement of the crack faces at the original crack tip location. The determination of CTOD in an SENB specimen is based on an assumption of rigid rotation as the specimen is loaded under bending, and that the specimen deforms about a plastic hinge ahead of the crack within the uncracked ligament. CTOD is then estimated by employing the similar triangles geometrical assumption, assuming a fixed location of the rotational point. By measuring the displacement at the crack mouth or a clip gauge at a given height above the crack mouth, the displacement at the crack tip (CTOD) can be determined. This approach has been adopted from the very earliest standards, and remains in BS 7448 to this day and in ISO 12135 for single point CTOD.

Critics of this approach point to uncertainties in defining the actual point of rotation in an SENB specimen. This is usually defined as being at a distance ahead of the crack tip equal to a proportion of the remaining ligament, and the proportion is called the rotational factor, $r_{p}$. BS 7448 assumes $r_{p}$ equals 0.4 for SENB specimens. Standard methods assume $r_{p}$ is a constant for the type of specimen, however, if the rotational factor is not actually constant then the use of a fixed rotational factor could introduce unnecessary errors for the estimation of CTOD. If the actual rotational factor is less than 0.4, CTOD is actually lower than that predicted with $r_{p}=0.4$ (i.e. BS 7448 would overestimate the actual CTOD); and conversely if the actual rotational factor is greater than 0.4 , assuming $r_{p}$ is 0.4 will give under-estimations of CTOD.

The actual rotational factor can be determined from the displacements from a double clip gauge (two clip gauge displacements $V_{1}$ and $V_{2}$ measuring at knife edge heights $z_{1}$ and $\mathrm{z}_{2}$ ), using the principles of similar triangles, to evaluate the assumptions about the definitions of $r_{p}$ using equation 8 . This was used to compare the predicted and actual location of the 
plastic hinge under loading of SENB specimens in three different strain hardening materials in this work.

$$
\mathrm{r}_{\mathrm{p}}=\left[\left(\frac{\mathrm{z}_{2}-\mathrm{z}_{1}}{\mathrm{~V}_{\mathrm{g} 2} / \mathrm{v}_{\mathrm{g} 1}-1}\right)-\left(\mathrm{z}_{1}+\mathrm{a}_{0}\right)\right] \times \frac{1}{\mathrm{~B}_{0}}
$$

\section{EFFECT OF STRAIN HARDENING ON CTOD}

\section{Japanese Equation}

Much of the historical development work for the CTOD testing standards was based on high strength and structural steels, which show relatively high ratios of yield strength to ultimate tensile strength $(\mathrm{Y} / \mathrm{T})$, typically 0.7 up to 0.95 . The standard CTOD equations in BS 7448 have been known to be less accurate for higher strain hardening material with lower $\mathrm{Y} / \mathrm{T}$ ratios, such as stainless steels [6,7]. To address this, research has been done to support the Japanese Welding Society in their development of a new rigid rotation equation for single value CTOD (now adopted into WES 1108) which accommodates the strain hardening properties individually within both the elastic and plastic components of CTOD [8,9];

$\delta=K^{2} \frac{\left(1-v^{2}\right)}{m_{J W E S} \sigma_{y s} E}+f_{p} \frac{0.43 B_{o} V_{p}}{0.43 B_{o}+a_{0}+z}$

Where

$\mathrm{m}_{\mathrm{JWES}}=4.9-3.5\left(\sigma_{\mathrm{ys}} / \sigma_{\mathrm{uts}}\right)$

Eq. 10

and $f_{p}$ is calculated by multiplying together a function of thickness, $\mathrm{f}(\mathrm{B})$, and a function of yield to tensile ratio, $\mathrm{f}\left(\sigma_{\mathrm{ys}} / \sigma_{\mathrm{uts}}\right)$, given respectively as;
$f(B)=0.8+0.2 \exp \{-0.019(B-25)\}$
Eq. 11
$\mathrm{f}\left(\sigma_{\mathrm{ys}} / \sigma_{\mathrm{uts}}\right)=-1.4\left(\sigma_{\mathrm{ys}} / \sigma_{\mathrm{uts}}\right)^{2}+2.8\left(\sigma_{\mathrm{ys}} / \sigma_{\mathrm{uts}}\right)-0.35$
Eq. 12

This method to include the material yield to tensile ratio $(\mathrm{Y} / \mathrm{T}$ ratio) within a rigid rotation approach to determine CTOD gives improved accuracy for single point values of CTOD $[7,10]$. However, it has not been developed for, or applied to CTOD tearing resistance curves before now.

\section{SENB notch silicone replicas}

The effect of the yield to tensile ratio on CTOD was shown by a casting series silicone replicas from the notches of SENB specimens in steels with three different tensile $\mathrm{Y} / \mathrm{T}$ ratios. The stress-strain curves from these three steels are shown in Fig. 1, and are summarized;

- M01 was a grade SA-543-GrB-Cl1 high strength steel with yield strength of $850 \mathrm{MPa}$. This had the highest $\mathrm{Y} / \mathrm{T}$ ratio of 0.93 .

- M02 was a grade S355J2 structural steel with yield strength of $421 \mathrm{MPa}$, and had a medium $\mathrm{Y} / \mathrm{T}$ ratio of 0.72 .
- M03 was grade SS316 austenitic stainless steels. Its yield strength was $286 \mathrm{MPa}$, giving it the lowest $\mathrm{Y} / \mathrm{T}$ ratio of 0.48 .

Replicas were cast at successive levels of applied load, based on increments determined from the load-displacement traces from equivalent standard single point tests in each material. By taking sections through the middle of each replica the value of CTOD could be measured (Fig. 2), and taken as the baseline for comparison to standard methods. The value of crack extension, $\Delta \mathrm{a}$, could also be measured and used to generate a CTOD R-curve for each specimen.

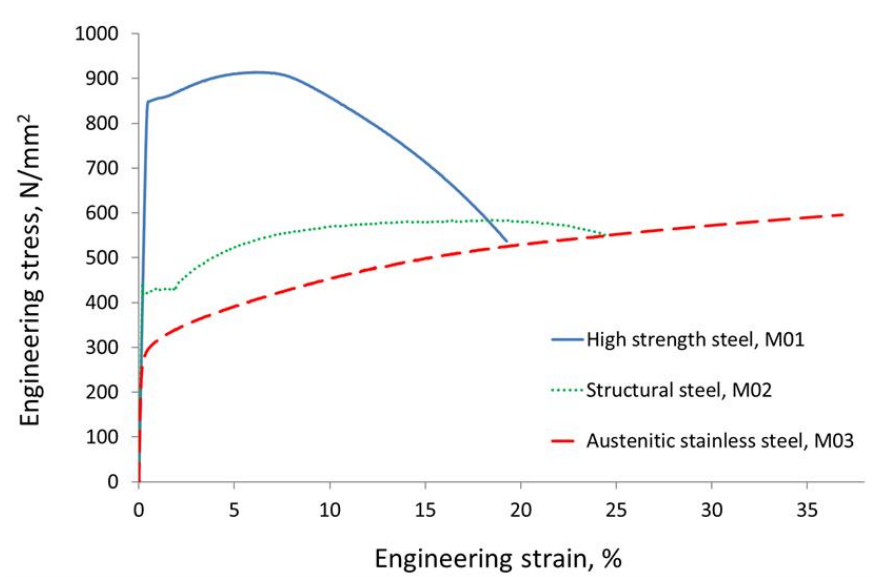

Figure 1 Stress versus strain curves for the three different steels tested in this work, identified as M01, M02 and M03 for the high Y/T ratio down to the lowest respectively.

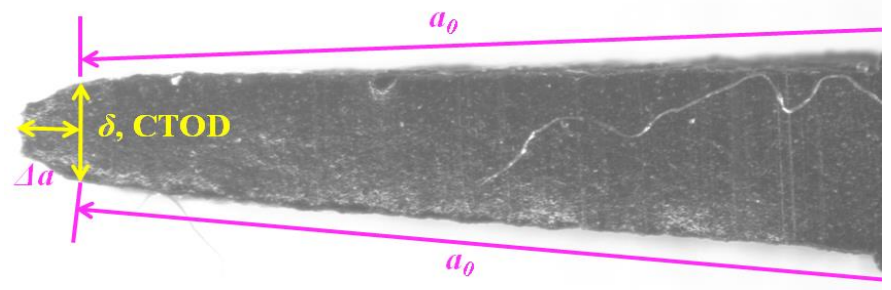

Figure 2 Section through the middle of a silicone replica of the notch cast from an SENB specimen during a fracture toughness test. The location for determination of CTOD, and the value of crack extension, $\Delta a$, for the plotting of the R-curve, are shown. Copyright TWI Ltd.

The effect of the yield to tensile ratio from the three steels on tearing can be seen qualitatively in Fig. 3, where the medium and high tensile ratio steels show the initiation of stable tearing after moderate levels of CTOD, the low tensile ratio stainless steel continues to blunt to high values of CTOD before tearing finally initiates. This difference in the crack tip behaviour under loading with different strain hardening materials is the reason that CTOD $\mathrm{R}$-curve formulae need to include the material tensile properties as variables. 

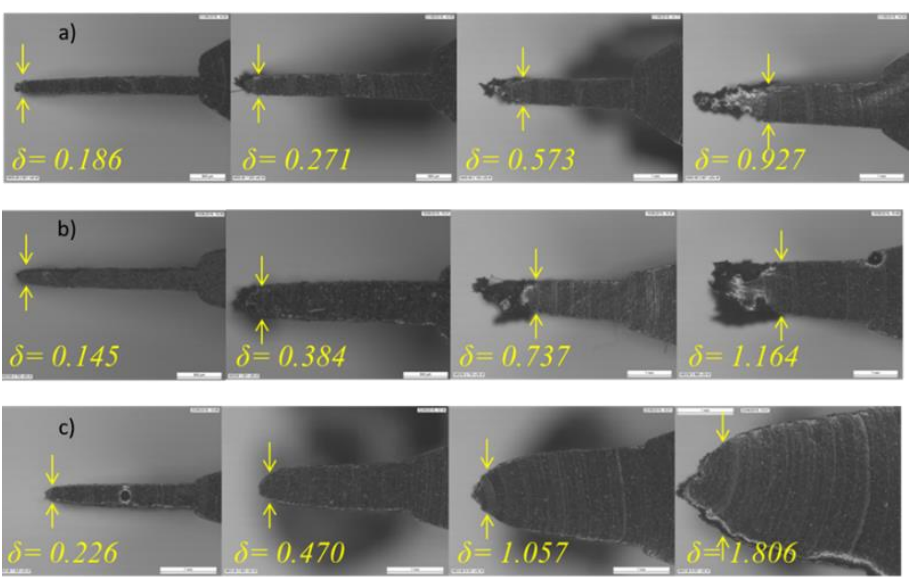

Figure 3 Sections through silicone replica casts taken at incremental stages through a fracture toughness test, for three different strain hardening materials, showing CTOD, $\delta$, in $\mathrm{mm}$; a) M01, high tensile ratio high strength steel; b) M02, medium tensile ratio structural steel; c) M03, low tensile ratio stainless steel. Copyright TWI Ltd.

\section{EVALUATION OF STANDARD METHODS FOR CTOD R- CURVES}

The measured values of crack extension from the silicone replica sections were used to plot CTOD R-curves for the replica measurements of CTOD, and for CTOD values determined according to standards BS 7448-4, ISO 12135 and ASTM E1820. The Japanese WES 1108 CTOD equation was also used as a comparison (identified as the JWES formula), but that CTOD method does not include an allowance for crack extension like the other standard R-curve methods. The CTOD R-curves for the three different materials are shown in Figs. 4 to 6 for the high $\mathrm{Y} / \mathrm{T}$ ratio to low $\mathrm{Y} / \mathrm{T}$ ratio steels respectively.

The J-based CTOD methods, ASTM E1820 and ISO 12135 , both significantly under-estimated the R-curves across all three materials. The BS 7448-4 formula was most accurate for the medium tensile ratio steel, under-estimated the highest tensile ratio, and over-estimated the lowest tensile ratio steel behaviour. The JWES formula, although not intended to be applied to the plotting of R-curves, gave the most consistent behaviour of slight under-estimation of the R-curves for high and medium tensile ratio steel, but was also under-estimating the Rcurve for the low tensile ratio stainless steel. These results highlight the lack of an approach to determine CTOD R-curves which gives sufficient accuracy for a range of strain hardening behaviour.

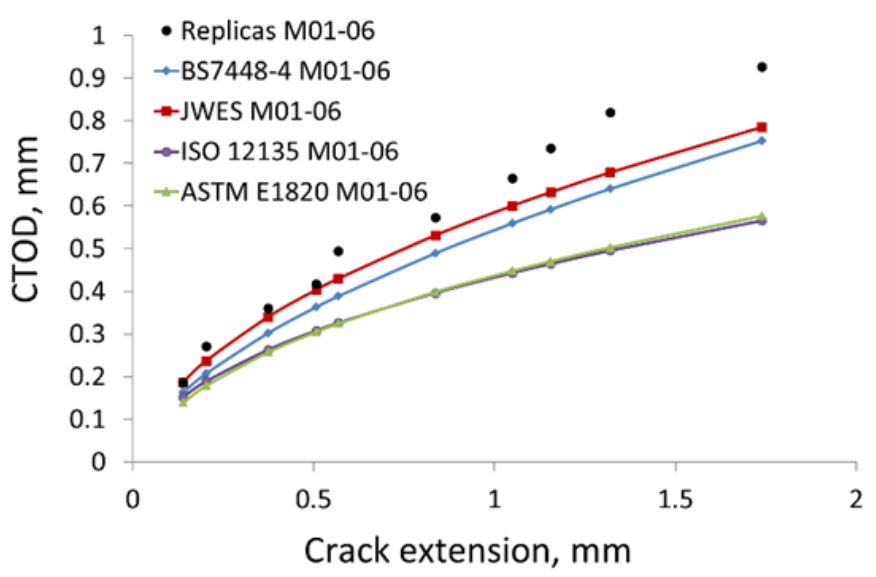

Figure 4 CTOD R-curves plotted from silicone replica measurements, and standard CTOD equations for high $\mathrm{Y} / \mathrm{T}$ ratio of 0.93 high strength steel M01.

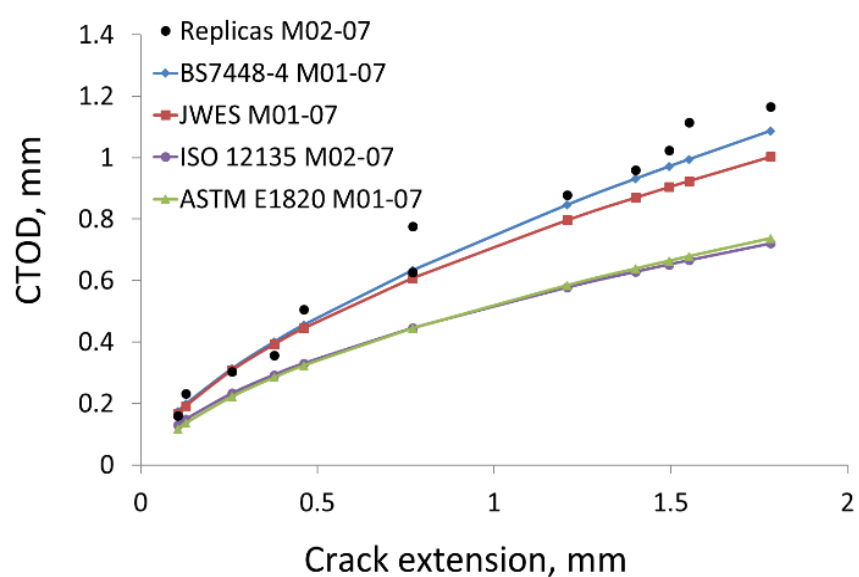

Figure 5 CTOD R-curves plotted from silicone replica measurements, and standard CTOD equations for medium $\mathrm{Y} / \mathrm{T}$ ratio of 0.72 structural steel M02.

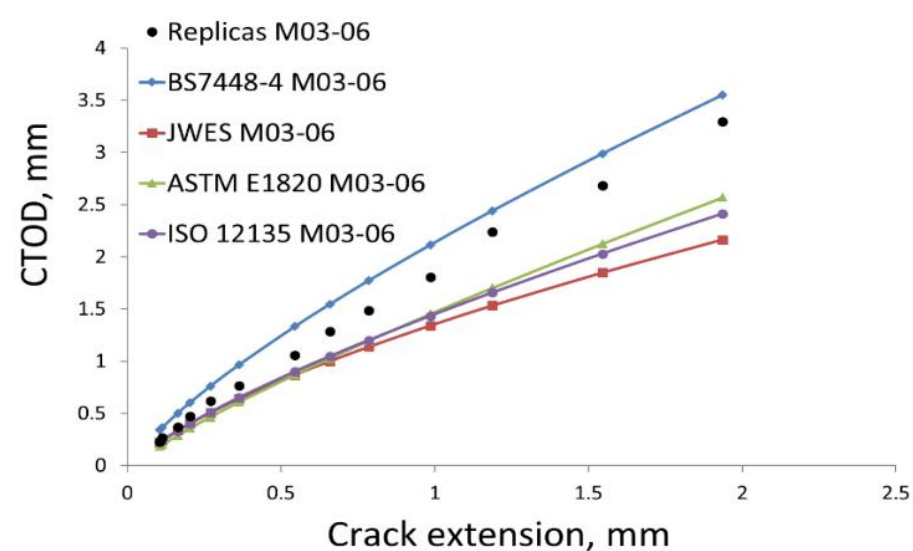

Figure 6 CTOD R-curves plotted from silicone replica measurements, and standard CTOD equations for low $\mathrm{Y} / \mathrm{T}$ ratio of 0.48 stainless stee M03. 


\section{NUMERICAL MODELLING OF RIGID ROTATION SENB SPECIMENS}

A method to determine CTOD R-curves using a rigid rotation assumption, while accounting for strain hardening was sought in the second part of this research. Experimentally, when Equation 8 was applied to the double clip data from the standard SENB tests, the value of the rotational point stabilised between 0.42 and 0.45 during the test in the medium $\mathrm{Y} / \mathrm{T}$ ratio steel, while $r_{p}$ settled at a value around 0.35 in the stainless steel. This suggested that $r_{p}$ was not constant for all materials, and could be a function of the $\mathrm{Y} / \mathrm{T}$ ratio. Therefore, a series of numerical models were generated to determine the effect of tensile behaviour on the rotational point. Abaqus software was used to generate models of SENB specimens equivalent to the dimensions of the experimental tests, modelled under 3-point bend loading [10], and the apex of the crack face opening was identified as the hinge location. Values of the CMOD and the node below the CMOD, CMOD-1 were extracted to evaluate $r_{p}$ based on the similar triangles method. The distance between the two nodes, $\Delta \mathrm{z}$ was found to be constant throughout the loading, therefore the nodes CMOD and CMOD-1 were used to give a good representation of the crack face angle. A value of $r_{p}$ was calculated based on Equation 8 modified to accommodate the model geometry, given as;

$r_{p}=\left(\frac{\Delta z}{\left(\frac{C M O D}{C M O D_{-1}}-1\right)}-\left(a_{0}-\Delta z\right)\right) \times \frac{1}{B_{0}} \quad$ Eq. 13

The values of $r_{p}$ from the models, plotted for CTOD between 0.2 and $1.0 \mathrm{~mm}$ for a range of tensile ratios $\left(\sigma_{\mathrm{ys}} / \sigma_{\mathrm{uts}}\right)$ is given in Fig. 7. It can be seen that the rotational factor changes with the material yield to tensile ratio.

A new strain hardening function of rotational factor, $r_{p}$ $s h$, was defined as a linear fit to the average of the numerical model $r_{p}$ data plotted in a linear relationship with the tensile ratio, and is described as:

$r_{p s h}=0.4668 \frac{\sigma_{y s}}{\sigma_{u t s}}+0.0996$

Eq. 14

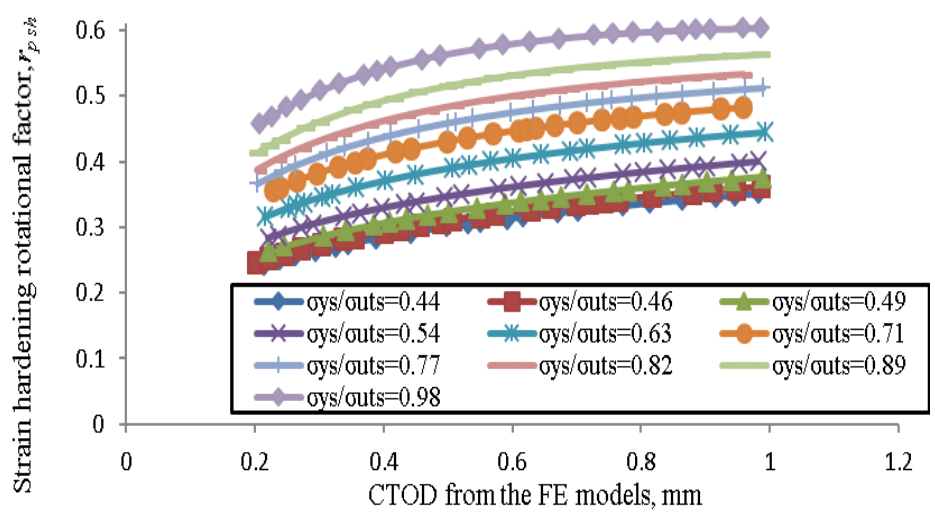

Figure 7 Values of the rotational factor, rp extracted from models of SENB specimens with different yield to tensile ratios.

\section{EVALUATION OF THE STRAIN HARDENING ROTATIONAL FUNCTION $r_{p}$ sh IN STANDARD CTOD FORMULAE}

The new strain hardening rotational function, $r_{p}$ sh developed from the numerical modelling was used to define two alternative replacements for the rigid rotation method currently given in BS 7448-4. The first method simply substituted $r_{p}$ sh and (1- $\left.r_{p ~ s h}\right)$ for the factors of 0.4 and 0.6 respectively in the plastic component of CTOD given in Equation 1. This first modified equation is given as Equation 15. The second approach was to also include a modification for the $\mathrm{Y} / \mathrm{T}$ ratio of the steel within the elastic component of CTOD as well as the plastic component, and was modified replacing the value of 2 with $\mathrm{m}_{\text {JWES }}$ (defined in Equation 10). The second modified equation is given in Equation 16.

$$
\begin{aligned}
& \delta=K^{2} \frac{\left(1-v^{2}\right)}{2 \sigma_{y s} E}+\frac{\left(1-r_{p s h}\right) \Delta a+r_{p s h} B_{o} V_{p}}{\left(1-r_{p s h}\right) \Delta a+r_{p} B_{o}+a_{0}+z} \quad \text { Eq. } 15 \\
& \delta=K^{2} \frac{\left(1-v^{2}\right)}{m_{J W E S} \sigma_{y s} E}+\frac{\left(1-r_{p s h}\right) \Delta a+r_{p s h} B_{o} V_{p}}{\left(1-r_{p s h}\right) \Delta a+r_{p} B_{o}+a_{0}+z}
\end{aligned}
$$

These modified CTOD R-curve equations were then used to analyse the fracture toughness test data from which the replicas had been obtained. These modified CTOD R-curves are plotted against the replica CTOD R-curves, and against BS 7448-4 and the JWES formulae again for comparison, in Figures 8 to 10 for high $\mathrm{Y} / \mathrm{T}$ ratio to low $\mathrm{Y} / \mathrm{T}$ ratio respectively.

\section{DISCUSSION}

An ideal equation is one that predicts CTOD as close as possible to the actual CTOD, as measured directly from a replica cast from the test specimen, while giving a consistent level of accuracy across a range of strain hardening alloys. While recognising that no method will be $100 \%$ accurate, it is preferable that the ideal equation would slightly under-estimate rather than over-estimate the value of CTOD to ensure safety. The concern about the BS 7448-4 approach is the likelihood to slightly over-estimate CTOD for the lowest tensile ratio alloys. But the ASTM E1820 and ISO 12135:2016 methods were shown to give significant under-estimates of the CTOD R-curves in all alloys.

The application of a new rotational factor function (with the yield to tensile ratio as a variable) in place of the fixed value of rotational factor within the BS 7448 Part 4 CTOD equation along with using the JWES ' $m$ ' factor in the elastic CTOD provided a method that gives the closest predictor of the CTOD R-curve from the silicone replicas. 


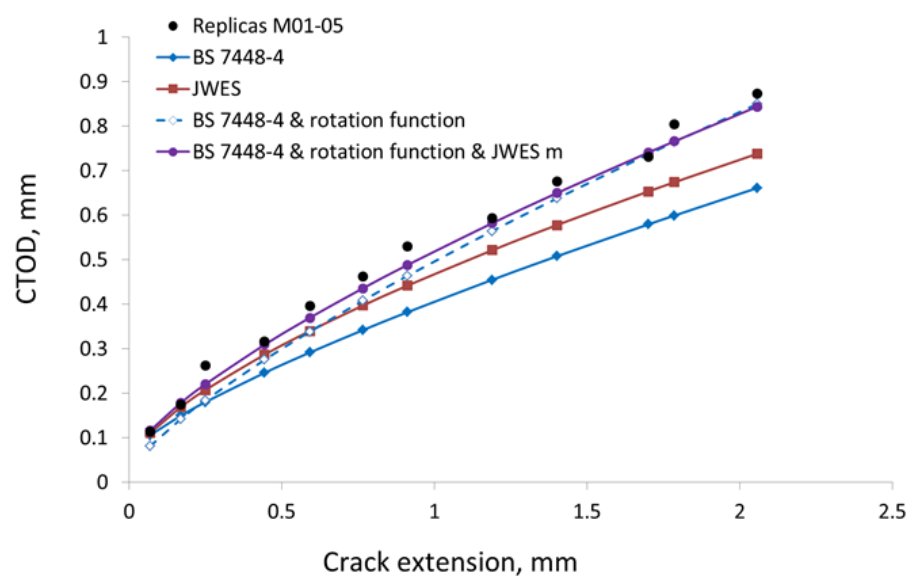

Figure 8 CTOD R-curves plotted from silicone replica measurements, and CTOD equations modified to account for strain hardening, for high $\mathrm{Y} / \mathrm{T}$ ratio of 0.93 high strength steel $\mathrm{M} 01$.

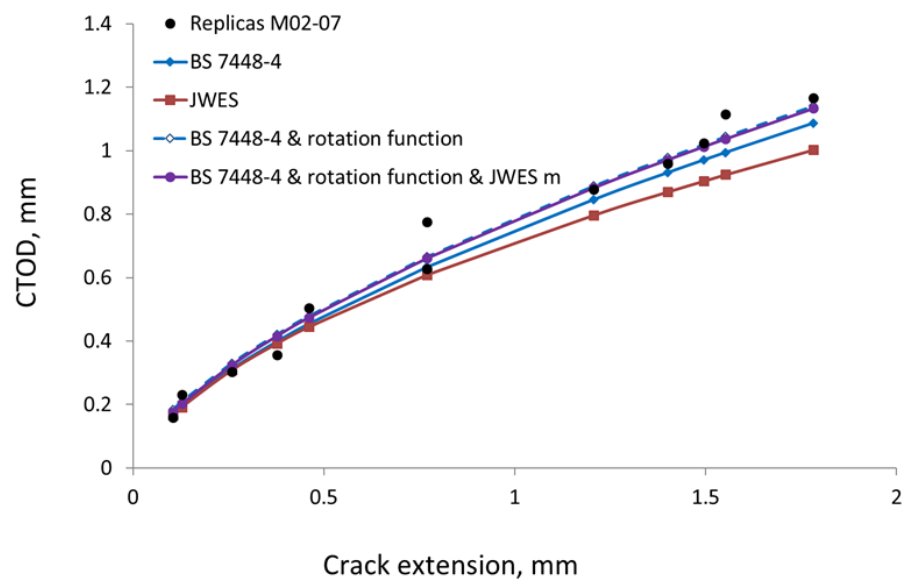

Figure 9 CTOD R-curves plotted from silicone replica measurements, and CTOD equations modified for strain hardening, for medium $\mathrm{Y} / \mathrm{T}$ ratio of 0.72 structural steel $\mathrm{M} 02$.

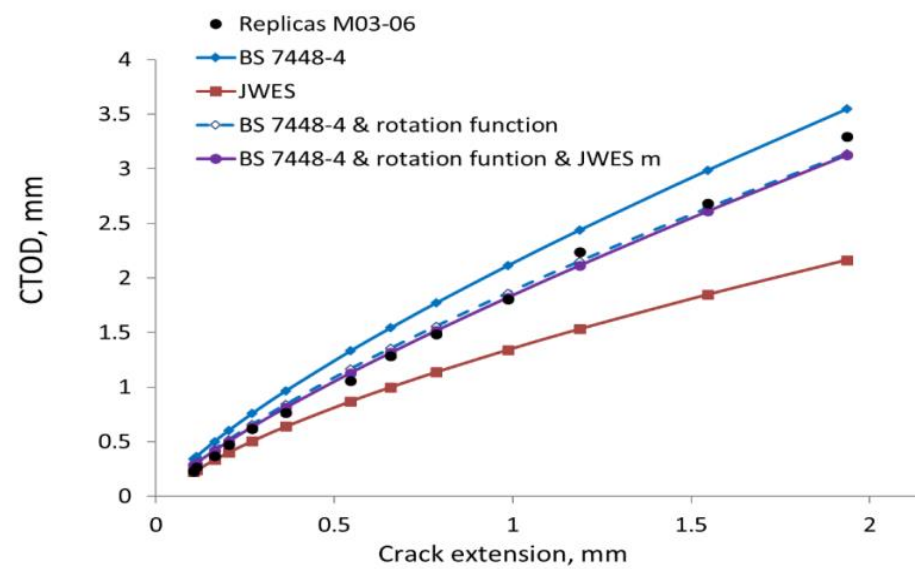

Figure 10 CTOD R-curves plotted from silicone replica measurements, and CTOD equations modified for strain hardening, for low $\mathrm{Y} / \mathrm{T}$ ratio of 0.48 stainless steel $\mathrm{M} 03$.
This work has investigated the CTOD determined from both rigid rotation assumption and the methods which define CTOD from J. There are practical reasons why each could be desirable. For instance, where clip gauges across the crack mouth are challenging (in very high speed, or environmental tests) being able to determine $\mathbf{J}$ from the load line displacement is a useful means to then define CTOD. But the comparison here shows that unless there is a reason to specifically prefer CTOD from J, then defining CTOD in terms of rigid rotation about a rotational point (corrected for strain hardening) is a more suitable approach, since it generally gives a more accurate value of CTOD without excessive conservatism, both from the JWES equation, and in the modified BS 7448-4 approach.

\section{CONCLUSIONS}

For CTOD R-curves, the methods based on CTOD from $\mathrm{J}$ in ISO 12135 and ASTM E1820 gave lower and less accurate R-curves than the rigid rotation methods in BS 7448-4 and WES 1108. However, the accuracy of the BS 7448-4 formula varied for the different materials, and over-estimated the Rcurves in low tensile ratio steel.

Investigating the effect of the rigid rotation assumption in different strain hardening materials led to a rotational factor function of tensile ratio, $r_{p}$ sh, to be developed from the results of numerical modelling work. When this function was substituted into standard equations in place of the fixed value of $r_{p}$ an improvement in the accuracy of BS 7448 Parts 1 and 4 was seen for the range of strain hardening investigated, especially when the elastic CTOD was modified to include the JWES ' $\mathrm{m}$ ' factor as well.

It is recommended that ISO 12135 returns to the use of a rigid rotation approach to determination of CTOD R-curves, provide a more accurate estimation of CTOD. A modified version of the current BS 7448-4 equation with the inclusion of $\mathrm{r}_{\mathrm{p} \mathrm{sh}}$ and $\mathrm{m}_{\text {JWES }}$ would ensure consistent and acceptable accuracy of CTOD R-curves for materials with tensile ratios from 0.44 to 0.98 .

The recommended CTOD R-curve equation for SENB specimens is therefore:

$$
\delta=K^{2} \frac{\left(1-v^{2}\right)}{m_{J W E S} \sigma_{y S} E}+\frac{\left(1-r_{p s h}\right) \Delta a+r_{p s h} B_{o} V_{p}}{\left(1-r_{p s h}\right) \Delta a+r_{p} B_{o}+a_{0}+z}
$$

\section{ACKNOWLEDGEMENTS}

Thanks The research presented here would not have been possible without the supervision of Chris Brown from Brunel University London, and by Dr. Henryk Pisarski, as part of TWI's NSIRC programme. The authors are grateful to Dan Bloom, Phillip Cossey, Jack Bradford and Jerry Godden for their contribution to performing the fracture toughness testing reported here. 


\section{REFERENCES}

[1] ISO 12135, 2016, "Metallic materials - Unified method of test for the determination of quasistatic fracture toughness", ISO.

[2] ASTM E1820, 2013, "Standard Measurement of Fracture Toughness", ASTM, 154.

[3] BS 7448-4, 1997, "Fracture mechanics toughness tests Part 4: Method for determination of fracture resistance curves and initiation values for stable crack extension in metallic materials". BSI, 1997.

[4] Zhu, X., 2009, "J-integral resistance curve testing and evaluation”, Journal of Zhejiang University SCIENCE A, 10(11), pp.1541-1560.

[5] Zhu, X.-K., Leis, B.N. \& Joyce, J., 2008, "Experimental Estimation of J-R curves from Load-CMOD Record for SE(B) Specimens", ASTM STP 1508, 5(5), pp.66-86.

[6] Wei, L., and Pisarski, H. G., 2007, "FEA investigations into the effects of geometry and tensile properties on J and CTOD in standard fracture specimens", ESIA9 - 9th International Conference on Engineering Structural Integrity Assessment, EMAS, Beijing, China, 15 - 19 October 2007.
[7] Khor, W, Moore P. L., Pisarski, H. G., Haslett, M., and Brown, C. J., 2016, "Measurement and prediction of CTOD in austenitic stainless steel", Fatigue \& Fracture of Engineering Materials \& Structures. 39: 1433-1442.

[8] Kawabata, T., Tagawa, T., Sakimoto, T., Kayamori, Y., Ohata, M., Yamashita, Y., Tamura, E., Yoshinari, H., Aihara, S., Minami, F., Mimura, H., and Hagihara, Y., 2016, "Proposal for a new CTOD calculation formula", Engineering Fracture Mechanics 159 (2016) 16-34.

[9] WES 1108, 2014, "Standard test method for Crack-Tip Opening Displacement (CTOD) fracture toughness measurement". The Japan Welding Engineering Society.

[10] Khor W, Moore P, Pisarski H and Brown C, 'Comparison of methods to determine CTOD for SENB specimens in different strain hardening alloys', 2018, in Fatigue \& Fracture of Engineering Materials and Structures (FFEMS), 41 (3), March 2018, pp 551-564. 
Copyright $@ 2018$ by ASME 